\title{
Photometric Variations of Solar Type Stars
}

\author{
By RICHARD R. RA DICK
}

Air Force Phillips Laboratory, Solar Research Branch, Sunspot, NM 88349, USA

High precision measurements of photometric variability among solar type stars have now been made since 1980. These observations clearly show that year-to-year brightness variations connected with magnetic activity are a widespread phenomenon among such stars. They also suggest that the Sun's potential for long-term white light variability may be significantly understated by measurements of solar total irradiance during the $1980 \mathrm{~s}$.

\section{Introduction: solar activity and variability}

Because our experience with the Sun so strongly influences our thinking about stellar behavior, it seems appropriate to begin this discussion of variability among solar type stars with a brief review of its solar paradigm. Our immediate attention will be focused on global properties of solar variability that can be (more or less) directly compared to analogous stellar behavior and used to help interpret it. In turn, our maturing stellar perspective may eventually provide a broader context for understanding and even predicting solar variability itself.

It is generally agreed that solar variability arises from the presence and evolution of magnetic fields on the Sun's surface. On the global scale, these magnetic fields are involved in the nonradiative heating of the Sun's chromosphere and corona. This nonradiative heating is designated by the term 'activity' (the term 'variability' refers more narrowly to the temporal behavior and, indirectly, to the spatial inhomogeneity of the activity). The chromosphere and corona get rid of their excess heat by radiating it away in specific spectral lines, such as the $\mathrm{H}$ - and K-lines of singly ionized calcium. Monochromatic pictures of the Sun in such lines (spectroheliograms) map the instantaneous distribution of the nonradiative heating, and presumably also faithfully map the underlying magnetic fields.

Clearly, we would like to have time series of magnetic flux measurements for both the Sun and its stellar analogues. Although such time series are available for the Sun, they are extremely difficult to generate for stars. Therefore, we are forced to resort to proxies, such as the chromospheric $\mathrm{Ca}$ emission index, which are accessible to stellar observations. It is important, however, to recognize that any proxy is likely to be imperfect. For example, the chromospheric Ca emission index suffers from two distinct defects: (1) It is sensitive to non-magnetic effects, such as acoustic heating and abundance differences, but (2) It is relatively insensitive to the magnetic flux contained in sunspots.

The global solar Ca K-line emission comprises the sum of two components - emission from the network, and emission from active region plages. The plage component increases dramatically from solar minimum to solar maximum, whereas the variation of the network component is less pronounced. The Sun's global white light variations are more complex. The conventional picture invokes three components; network faculae, active region faculae, and dark sunspots, with the net variation representing the competitive balance between the bright faculae and the dark spots (e.g., Foukal \& Lean 1988). On the time scale of the 11-yr solar activity cycle, roughly half of the total positive contribution from the faculae is cancelled by the opposing flux deficit from the sunspots, leaving a net white light variation that approximately tracks the cyclic variation of solar activity as measured, for example, by the global $\mathrm{Ca} \mathrm{K}$-line emission. 
By appropriately suppressing spatial resolution, a time series of disk integrated solar fluxes may be created. Such time series are available for both $\mathrm{Ca} \mathrm{K}$-line emission (from the National Solar Observatory: Keil 1992; Livingston 1992) and white light total irradiance (from SMM/ACRIM or NIMBUS-7/ERB), and provide solar data that may be compared to stellar observations with relative ease.

\section{Stellar activity and variability}

\subsection{The co-ordinated Lowell and Mount Wilson programs}

Modern synoptic measurements of photometric variability among solar type stars have been made since 1980, when observations of lower main sequence stars in the Hyades, a nearby cluster $10-15 \%$ the age of the Sun, were inaugurated at the Cloudcroft Observatory (Radick 1991 and references therein). This program was moved to the Lowell Observatory in 1982, where photometric monitoring of a few Hyades stars continues up to the present time. Between 1984 and 1987, stars in the Hyades-age Coma Berenices cluster were added. The heart of the Lowell photometric program, however, traces back to 1984, when regular observations of 33 field stars, selected from among those of the Mount Wilson HK Project, were begun (Lockwood 1994). The HK Project itself can be traced back to 1966 , when regular spectrophotometric observations of chromospheric $\mathrm{Ca} \mathrm{H}+\mathrm{K}$ emission from about 100 stars began at the Mount Wilson Observatory (Wilson 1978). All but two of the 33 stars of the Lowell program are among these HK Project 'veterans.'

The Lowell and Mount Wilson observations demonstrate that both broadband and chromospheric $\mathrm{Ca} \mathrm{H}+\mathrm{K}$ emission variations are common among solar type stars. The time scale of variation ranges from the night-to-night interval between the individual observations to the present 27 year duration of the HK Project. The amplitude of variation shows the dependences on stellar mass (or temperature) and rotation rate (or age) predicted by stellar dynamo theory (e.g., Radick 1992). Cyclic variability reminiscent of the 11-yr solar activity cycle, however, appears to be confined largely, if not exclusively, to the older, solar-age stars.

Although photometric brightness and $\mathrm{Ca} \mathrm{H}+\mathrm{K}$ emission variations are clearly connected phenomena, their relationship is neither simple nor accurately predictable. Among young stars, the year-to-year brightness and $\mathrm{H}+\mathrm{K}$ emission variations are anti-correlated, whereas for the older, solar-age stars, they tend to correlate directly, as they do for the Sun. Although the Sun's chromospheric K-line emission variation appeared to be normal during the 1980's, the amplitude of its white light variation seems to have been distinctly less than the variation of otherwise similar stars (Radick et al. 1990; Lockwood et al. 1992).

\subsection{Some specific solar-stellar comparisons}

Figure 1 displays the time series data for the Sun and two stars, HD10476 and HD1835. Although it is somewhat cooler and less massive than the Sun, HD10476 (= HR493, $B--V=0.84, S p=K 1 V$ ) has an average level of chromospheric activity, as measured by $\mathrm{Ca} \mathrm{H}+\mathrm{K}$ emission, that is very close to that of the Sun. Like the Sun, it is a slow rotator, with a period of about 35 days. These circumstances imply that it is comparable in age to the Sun. In contrast, HD1835 (= HR88, $B--V=0.66, S p=G 2 V)$ is a typical young star. It is a member of the Hyades group, which suggests that its age is about $10-15 \%$ that of the Sun. This is confirmed by the star's relatively rapid rotation (its period is about 8 days) and high average level of chromospheric activity (about three times the solar level). Its temperature and mass closely match those of the Sun.

The upper panels in Figure 1, which are all plotted with the same ordinate scale, 

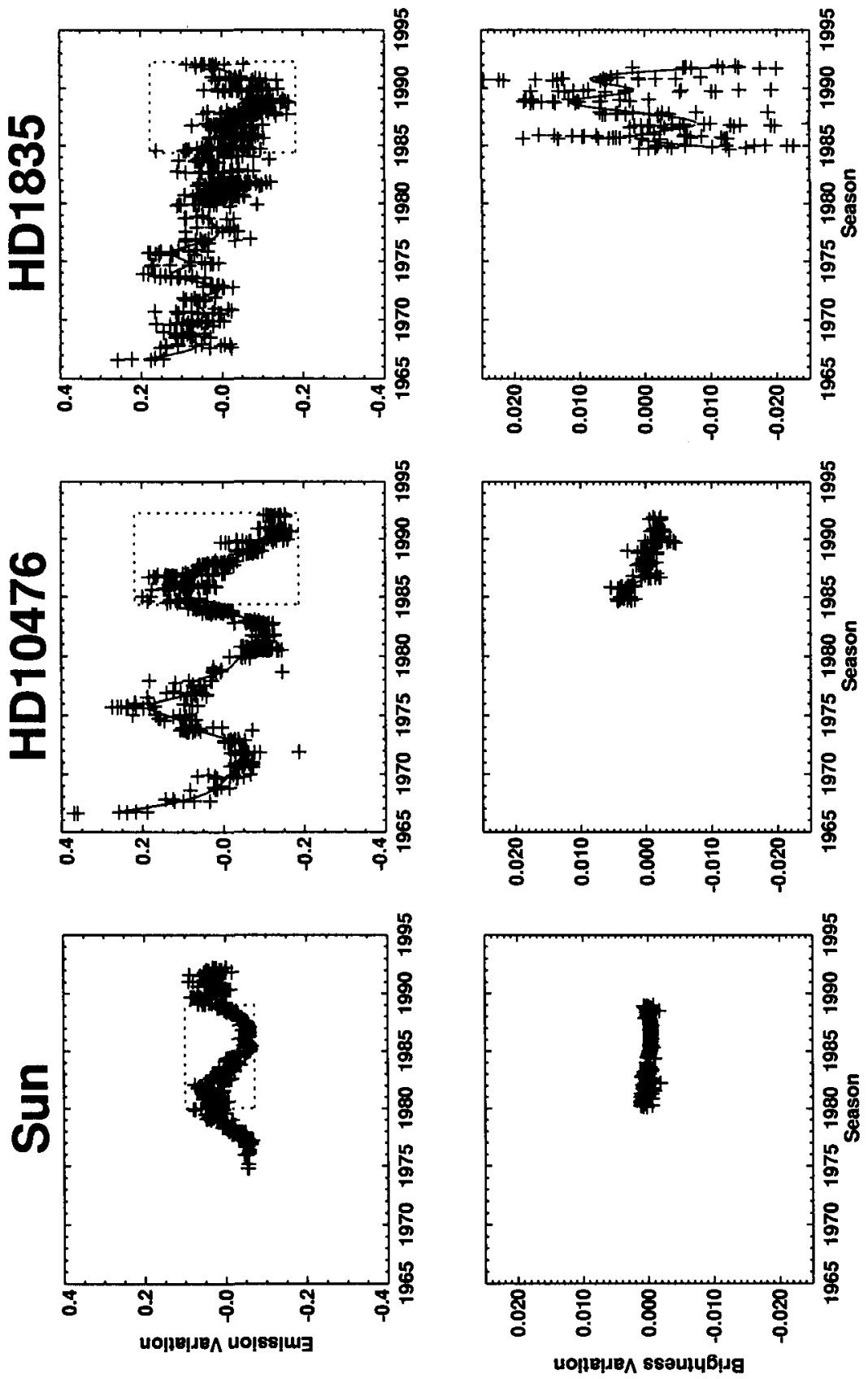

FIgURE 1. Time series data for the Sun and two stars

show the (fractional) variation in chromospheric emission. The lower panels, also plotted using a single ordinate scale, show the corresponding photometric variations. The box within each of the upper panels encloses the interval of the contemporaneous broadband observations shown below. Embedded in each time series is a cubic spline fitted to the annual mean values, which can be helpful for tracing out year-to-year fluctuations, 
especially for cases like the one shown in the lower right panel where the annual changes are masked by strong night-to-night variations. The solar data are the NSO K-index measurements (Livingston 1992) and the SMM/ACRIM radiometry (Willson 1992). The stellar data are from the Mount Wilson and Lowell Observatories.

Both the Sun and HD10476 show clear activity cycles approximately a decade in length. The amplitude of the solar cycle, however, is less than half that of HD10476. Like many young stars, HD1835 shows strong, random-looking variability, superimposed on a long-term secular trend (in this case, downward), but no obvious activity cycle.

The Sun's white light cyclic variation (lower left panel) is almost imperceptible on the plotted scale. In contrast, the photometric variation of HD10476, which is 5-6 times larger than that of the Sun, can clearly be seen to track the downward trend of its $\mathrm{Ca}$ $\mathrm{H}+\mathrm{K}$ emission. As mentioned previously, this correlated behavior is a hallmark of older, less active stars, including the Sun. This suggests that the cyclic white light variability of solar-age stars as a class is governed by bright features, as it is for the Sun. In contrast to either the Sun or HD10476, the photometric variations of HD1835 are very pronounced, on both the night-to-night and the year-to-year time scales. The variability amplitude amounts to $2-3 \%$, on both time scales. Furthermore, the photometric variations do not track the $\mathrm{H}+\mathrm{K}$ variations on the year-to-year time scale, but rather mirror them in an anticorrelated pattern that is characteristic of young stars. This suggests that, in contrast to solar-age stars, the year-to-year photometric variations of young stars are controlled by dark features.

When the year-to-year variation is suppressed, the relation between the $\mathrm{Ca} H+\mathrm{K}$ and broadband residuals (whenever it is statistically significant) is almost always found to be an anticorrelation, for solar-age stars as well as young stars. This suggests that (1) The surface features of solar type stars, both young and old, are consistent with active regions: dark spots temporally and spatially associated with bright plages, and (2) spots dominate short-term variation, for all stars.

Figure 1 illustrates two points: (1) It is not hard to find sunlike, solar-age stars (like HD10476) that vary more than the Sun, and (2) Chromospheric and broadband variations are not simply proportional, which raises a warning flag about the common practice of using one activity indicator as a proxy for another.

\subsection{Characteristics of the entire sample}

In Figure 2, we turn our attention to the entire sample of 33 stars. In the two panels of this diagram, chromospheric and photometric variability are plotted against average chromospheric activity. In both cases, variability is measured by the rms dispersion of annual mean values (in fractional units), and average activity ('chromospheric emission ratio') is measured relative to bolometric luminosity and plotted on a logarithmic scale. A point representing the Sun has also been plotted in each panel. In the lower panel, stars which are judged to be variable on the basis of the internal evidence of the photometry alone are indicated by asterisks, and stars whose variability cannot be clearly discerned relative to the measurement noise are designated by crosses.

The upper panel shows that the amplitude of $\mathrm{Ca} \mathrm{H}+\mathrm{K}$ emission variation increases with average activity, and that the Sun is near the center of the range in variability for stars of comparable average activity. The amplitude of photometric variation also increases with average activity, but more abruptly. Young, active stars almost always show strong photometric variability. Indeed, the lower panel of Figure 2 suggests that the young Sun probably varied by more than a percent from year to year, like HD1835 or even the star in the upper right corner of the diagram (HD129333, whose age is probably only a few percent that of the present Sun). Among stars comparable to the Sun in 

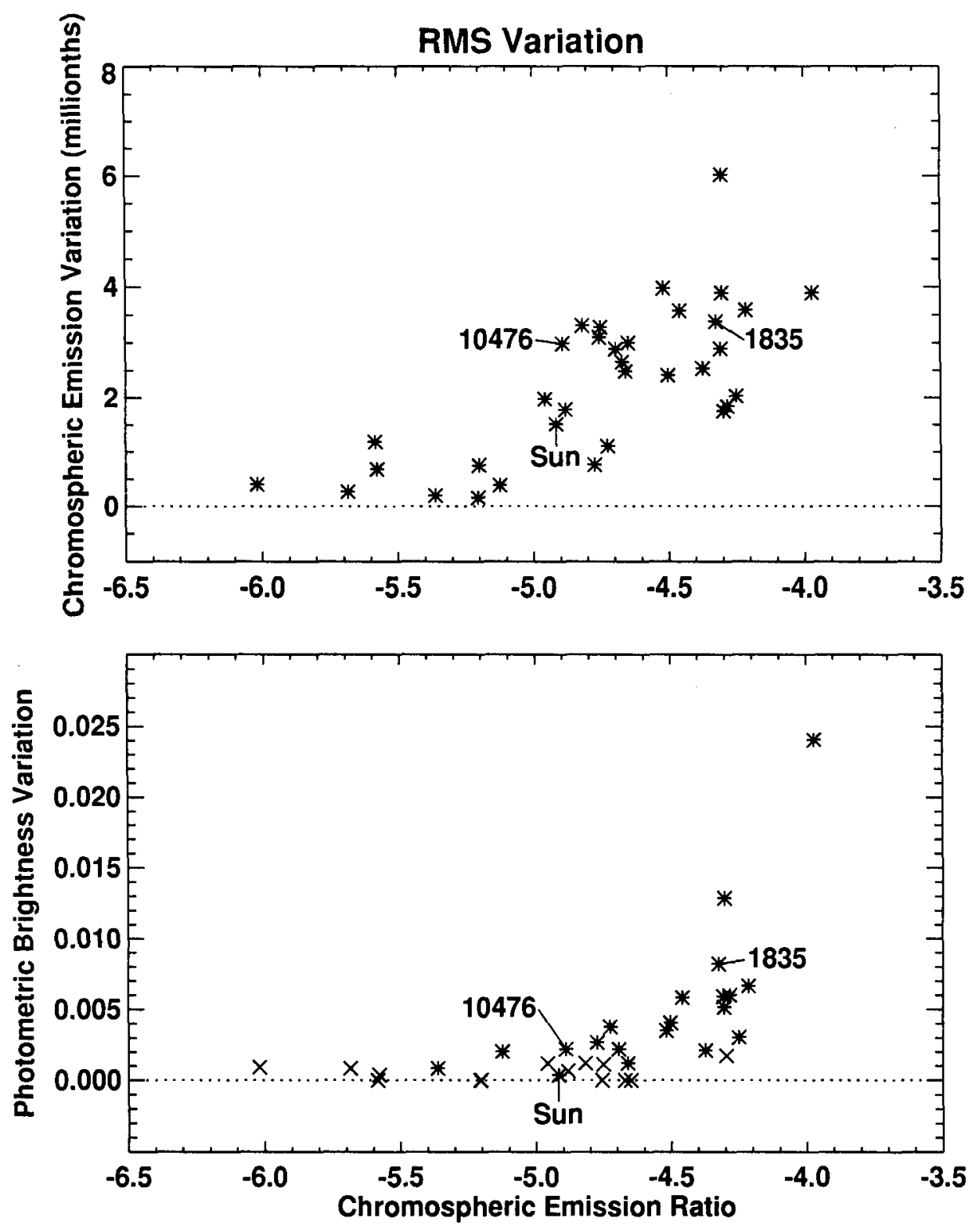

FIgURE 2. Cyclic chromospheric and photometric variability as a function of mean chromospheric activity.

average activity, about half vary, some by as much as about $0.5 \%$. Stars even less active than the Sun are only occasionally found to vary, and at relatively low amplitudes. It is interesting that the Sun appears to be near the bottom of the photometric distribution, even though its amplitude of $\mathrm{Ca} H+K$ variation seems normal. The Sun is not the only star for which the amplitudes of photometric and chromospheric variation are not very tightly coupled. In other words, chromospheric variation is not a particularly precise 
predictor for photometric variability. Likewise, average activity predicts photometric variability to only a factor of two or three.

\section{Analysis: calibration issues and other matters}

The surprisingly low white light variability shown by the Sun evokes concern that it might be an artifact. This, in turn, has led to a detailed and continuing examination of a long list of selection and calibration issues aimed at reducing this possibility. These tend to involve either (1) relating the solar to the stellar measurements, or (2) identifying and correcting systematic effects in the observations. The flavor of this process can perhaps be appreciated from a few examples.

The two NSO K-indices and the Mount Wilson S-index, all of which measure chromospheric $\mathrm{Ca}$ emission, are surprisingly difficult to relate in a satisfactory manner. Except for a handful of measurements made between late 1975 and early 1977 , there are no contemporaneous data presently available that directly link the $\mathrm{K}$-indices with the $(\mathrm{H}+\mathrm{K})$ $\mathrm{S}$-index, and the available overlapping observations are inadequate to properly constrain even a linear (i.e., two-parameter) regression. In order to sample a wider range of activity, Duncan et al. (1991) resorted to high dispersion spectrograms of the K-line region from Lick Observatory for 15 stars from the Mount Wilson HK Program. Seven of these 15 stars have only one or two measurements of the S-index, and none have more than 30 . The K-line spectrograms are all single plates. Because the chromospheric emission for several of the 15 stars is known to vary strongly and rapidly, and because none of the observations are simultaneous, it is virtually assured that the corresponding measurements for a good number of these stars sample different activity levels. Juggling difficulties such as these, a recent re-evaluation of the calibration led to the relation: $S=1.48 * K+0.041$, with standard errors of 0.07 and 0.013 in the slope and intercept, respectively (the original calibration yielded $S=1.54 * K+0.040$, with no error estimates). The bottom line of all this is that the uncertainty in the value for the average chromospheric emission ratio (the abscissa of Figure 2) for the Sun amounts to about $20 \%$.

If anything, the issues relating to the calibration and correction of the photometric observations can be even more vexing (Lockwood 1993). Besides the noise arising from sources such as scintillation, counting statistics, sky transparency fluctuations, and centering and guiding errors, attention must be given to effects such as variability among the photometric reference stars and long-term, secular drifts in the instrumental response.

The variability of the reference stars must be handled on a case by case basis. Fortunately, it is often relatively easy (at least, after a few years have elapsed!) to determine that a particular reference star is not stable, and even to estimate with some accuracy its contribution to the rms dispersions that we have adopted to measure variability. Typically, the contribution to the composite dispersion (calculated from annual mean values) from reference star variability is about $\mathbf{0 . 0 0 0 6}$, and ranges from 0.0003 to 0.0029 .

Like reference star variability, secular drifts become apparent only after the fact. For the Lowell photometry, the principal effect appears to be a gradual drift in the color response of the photomultiplier tube that aliases brightness changes whenever there are color differences among the program and reference stars. Because a stable configuration for the Lowell instrumentation has been deliberately maintained, it has been possible to use observations dating back as far as 1973 to trace and calibrate this effect. For the present measurements, its contribution to the composite relative dispersion is typically 0.0002 , and ranges from 0.0000 to 0.0010 .

Another issue involves the relation between variability measured in a finite bandpass (the Lowell photometry, here the sum of measurements made in Strömgen $b$ and $y$ fil- 
ters centered at 472 and $551 \mathrm{~nm}$, respectively) and variability in total irradiance (the $\mathrm{SMM} / \mathrm{ACRIM}$ solar radiometry, which is effectively bolometric). Were the Sun to radiate as a black body, it is not hard to estimate that its Strömgen $b+y$ variation would be $25-30 \%$ larger than its bolometric variation. When scaled by this factor, the rms variability of the Sun becomes 0.0004 (on the Lowell system). However, the solar total irradiance is known to be characterized by substantial excess variation in the extreme UV, relative to the amount predicted by the black body model. Because this EUV excess must be counterbalanced elsewhere, the true correction is probably smaller than the factor of $25-30 \%$ stipulated by the black body model, but by how much is not clear.

\section{Summary}

Available time series data now permit meaningful comparisons of the variability of the Sun and its stellar analogues on time scales approximating that of the solar activity cycle. When this is done, some reassuring patterns emerge. Among the more notable of these is the indisputable tendency for solar-age stars, including the Sun, to show cyclic white-light variations that approximately track the corresponding activity fluctuations. However, the amplitude of the Sun's white light variation, as measured during the 1980s, seemed to be significantly less than that of many of its stellar analogues, despite that fact that the amplitude of the Sun's chromospheric emission variation was entirely typical for a star of its type. Although the relation between the broadband observations for the Sun and its stellar analogues is subject to several adjustments which tend to narrow this discrepancy (from perhaps a factor of eight, for the uncorrected data, to about a factor of four), they do not eliminate it. Thus, if this result is accepted at face value, it implies that the coupling between photometric and chromospheric variations is not very tight, raising a warning about the casual use of one variability indicator as a proxy for another.

Acknowledgments. This work was supported by the Air Force Office of Scientific Research under Task 2311G3.

\section{REFERENCES}

Duncan, D.K., Vaughan, A.H., Wilson, O.C., Preston, G.W., Frazer, J., Lanning, H., Misch, A., Mueller, J., Soyumer, D., Woodard, L., Baliunas, S.l., Noyes, R.W., Hartmann, L.W., Porter, A., Zwaan, C., Middelkoop, F., Rutten, R.G.M. \& Mrhalas, D. $1991 \mathrm{Ca}$ II H and K measurements made at Mount Wilson Observatory, 1966-1983. Astrophys. J. Suppl. 76, 383-430.

Foukal, P.V. \& LEAN, J. 1988 Magnetic modulation of solar luminosity by photospheric activity. Astrophys. J. 328 347-357.

KEIL, S.L. 1992 Private communication.

Livingston, W.C. 1992 Private communication.

Lockwood, G.W. 1994 Irradiance Observations of Stars. In The Sun as a Variable Star: Solar and Stellar Irradiance Variations (ed. J.M. Pap, C. Fröhlich, H.S. Hudson, \& S.K. Solanki). Cambridge University Press, in press.

Lockwood, G.W., Skiff, B.A., Baliunas, S.L. \& Radick, R.R. 1992 Long-term solar brightness changes estimated from a survey of Sun-like stars. Nature 360, 653-655.

Radick, R.R. 1991 The luminosity variability of solar-type stars. In The Sun in Time (ed. C.P. Sonett, M.S. Giampapa, \& M.S. Matthews). pp. 787-808. Univ. of Arizona Press, Tucson, AZ, USA.

RADICK, R.R. 1992 Luminosity variability of lower main-sequence stars. In The Solar Cycle (ed. K.L. Harvey). Astronomical Society of the Pacific Conference Series 27, pp. 450-464. 
Radick, R.R., Lockwood, G.W. \& Baliunas, S.L. 1990 Stellar activity and brightness variations: a glimpse at the Sun's history. Science 247, 39-44.

Wilson, O.C. 1978 Chromospheric variations in main-sequence stars. Astrophys. J. 226, 379396.

WILLSON, R.C. 1992 Private communication. 